\title{
DIFFERENTIAL PHASE-CONTRAST X-RAY COMPUTED TOMOGRAPHY: FROM MODEL DISCRETIZATION TO IMAGE RECONSTRUCTION
}

\author{
Masih Nilchian and Michael Unser
}

Biomedical Imaging Group, EPFL, Switzerland

\begin{abstract}
Our contribution in this paper is two fold. First, we propose a novel discretization of the forward model for differential phase-contrast imaging that uses B-spline basis functions. The approach yields a fast and accurate algorithm for implementing the forward model, which is based on the first derivative of the Radon transform. Second, as an alternative to the FBP-like approaches that are currently used in practice, we present an iterative reconstruction algorithm that remains more faithful to the data when the number of projections dwindles. Since the reconstruction is an ill-posed problem, we impose a total-variation (TV) regularization constraint. We propose to solve the reconstruction problem using the alternating direction method of multipliers (ADMM). A specificity of our system is the use of a preconditioner that improves the convergence rate of the linear solver in ADMM. Our experiments on test data suggest that our method can achieve the same quality as the standard direct reconstruction, while using only one-third of the projection data. We also find that the approach is much faster than the standard algorithms (ISTA and FISTA) that are typically used for solving linear inverse problems subject to the TV regularization constraint.
\end{abstract}

Keywords: differential phase-contrast imaging, alternating direction method of multipliers (ADMM), Radon transform, preconditioned conjugate gradient method, filtered back projection (FBP).

\section{INTRODUCTION}

Conventional X-ray computed tomography aims at reconstructing the distribution of the absorption coefficient of the object. However, many materials in biological and medical samples show low absorption contrast, while producing indicative phase shift in the transmitted X-ray beam [1]. The differential phase-contrast imaging (DPCI) is a recent modality that utilizes this phase information to reconstruct the distribution of the refractive index of the object.

The physical model of DPCI is based on the first derivative of the Radon transform (FDRT) [2]. The consequence is that images can be reconstructed using a variant of the filtered back projection (FBP) algorithm used in conventional tomography [2]. However, since the FBP requires a large number of projections, the imaging acquisition time is very long, on the order of hours. The situation is even worse (on the order of days) in neutron tomography which has the same mathematical model [3]. The key factor at the time of data acquisition is the number of view angles that will be later required to reconstruct the object. To lessen this number, it is natural to formulate the reconstruction as an inverse problem and to use prior information to reconstruct the object with an appropriate iterative algorithm.

This work was funded (in part) by the Center for Biomedical Imaging of the Geneva-Lausanne Universities and EPFL, as well as by the Foundations Leenaards and Louis-Jeannet.
The development of iterative methods for DPCI is very recent [4, 5] and there are several aspects that have not yet been optimized. The first concerns the implementation of the forward imaging model. Qi in [4] implemented the derivative of the Radon operator as a weighted sum of projections along the two object dimensions; this simplified scheme, however, performs poorly with noisy measurements. Kohler et al. use Kaiser-Bessel windows (isotropic blob functions) as basis functions whose Radon transform is known analytically and can be readily differentiated [5]. The downside of the approach is that the blob functions fail to satisfy the partition of unity, which is a necessary condition for controlling the error of approximation [6]. The second aspect is computational speed. The two methods proposed so far are first-order schemes whose convergence speed is at the low end of what has been achieved with other modalities.: the first uses a basic steepest decent algorithm [4], while the second relies on the Newton-Raphson method with SeparableParaboloidal-Surrogate [5].

In this paper, we propose a new discrete model for DPCI based on polynomial B-splines which are known to offer the best cost/quality tradeoff among interpolators. We show that the model lends itself to an analytical treatment and that it yields a fast and accurate implementation of the forward model. Based on this model, we then design an iterative reconstruction algorithm that imposes a combination of TV and Tikhonov regularization constraints. Our new method follows an augmented-Lagrangian optimization principle and makes use of the conjugate gradient method to solve the linear step in the alternating direction method of multipliers (ADMM). The use of a problem-specific preconditioner makes it significantly faster than competing algorithms.

The paper is organized as follows: in Section 2, we briefly review the mathematics of the Radon transform and derive the corresponding properties of the FDRT and its adjoint. We propose a discrete model for the FDRT together with a fast implementation in Section 3. In Section 4, we present our iterative reconstruction algorithm. We provide some simulation results in Section 5 to validate our method.

\section{RADON TRANSFORM AND ITS FIRST DERIVATIVE}

The Radon transform is an important mathematical tool in many applications of straight-ray tomography with complete projection data. In order to specify the operator, we need to set our 2-D geometry. We define the unit vector $\boldsymbol{\theta}=(\cos \theta, \sin \theta)$ orthogonal to the line of integration, as depicted in Figure 1. The spatial coordinates of the input function are denoted by $\boldsymbol{x}=\left(x_{1}, x_{2}\right)$. They are also expressed in a rotated coordinate system as $\boldsymbol{x}=y \boldsymbol{\theta}+t \boldsymbol{\theta}^{\perp}$, where $t \in \mathbb{R}$ and $\boldsymbol{\theta}^{\perp}=(-\sin \theta, \cos \theta)$ is the unit vector along the integral line.

Definition 1. [7] The (2-dimensional) Radon transform $\mathcal{R}$ : $L_{2}\left(\mathbb{R}^{2}\right) \longmapsto L_{2}(\mathbb{R} \times[0, \pi])$ maps a function on $\mathbb{R}^{2}$ into the set of its integrals over the lines of $\mathbb{R}^{2}$. Specifically, if $\boldsymbol{\theta}=(\cos \theta, \sin \theta)$ 


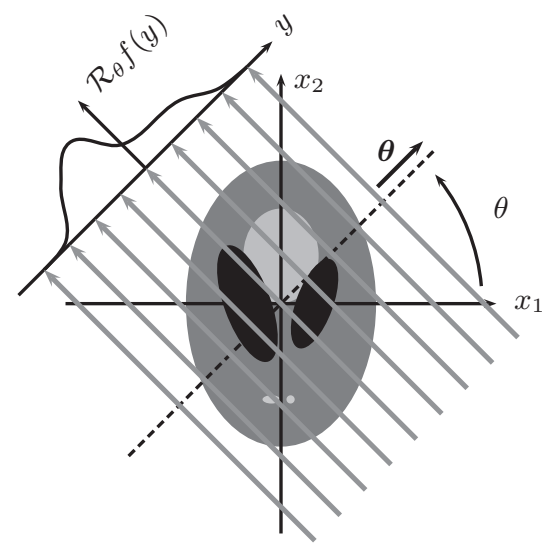

Fig. 1: The object lies in a 2-D plane and is imaged at an angle $\theta$.

and $y \in \mathbb{R}$, then

$$
\mathcal{R}\{f\}(y ; \theta)=\int_{\mathbb{R}^{2}} f(\boldsymbol{x}) \delta(y-\langle\boldsymbol{x}, \boldsymbol{\theta}\rangle) \mathrm{d} \boldsymbol{x}=\int_{\mathbb{R}} f\left(y \boldsymbol{\theta}+t \boldsymbol{\theta}^{\perp}\right) \mathrm{d} t .
$$

It represents the set of line integrals of $f \in L_{2}\left(\mathbb{R}^{2}\right)$ perpendicular to $\boldsymbol{\theta}$ with the (signed) distance $y$ from the origin. For brevity, $\mathcal{R}\{f\}(y ; \theta)$ is sometimes denoted by $\mathcal{R}_{\theta} f(y)$.

\subsection{Review of Standard Results}

The Radon transform is a linear operator with the following essential properties

- Pseudo-commuting with convolution

$$
\mathcal{R}_{\theta}\{f * g(\boldsymbol{x})\}(y)=\left(\mathcal{R}_{\theta} f * \mathcal{R}_{\theta} g\right)(y),
$$

- Projected translation invariance

$$
\mathcal{R}_{\theta}\left\{f\left(\cdot-\boldsymbol{x}_{0}\right)\right\}(y)=\mathcal{R}_{\theta} f\left(y-\left\langle\boldsymbol{x}_{0}, \boldsymbol{\theta}\right\rangle\right) .
$$

The adjoint of the Radon transform is an operator that maps back projection data into the space domain. It is given by

$$
\mathcal{R}^{*}\left\{g_{\theta}(y)\right\}(\boldsymbol{x})=\int_{0}^{\pi} g_{\theta}(\langle\boldsymbol{x}, \boldsymbol{\theta}\rangle) \mathrm{d} \theta,
$$

where $g_{\theta}(y) \in L_{2}(\mathbb{R} \times[0, \pi])$. The Radon adjoint of the Radon transform globally acts as a smoothing filter on the object $f(\boldsymbol{x})$; since

$$
\begin{aligned}
\mathcal{R}^{*} \mathcal{R}\{f\}(\boldsymbol{x}) & =\left(f * \frac{1}{\|\cdot\|}\right)(\boldsymbol{x}) \\
& =2 \pi \times(-\triangle)^{-\frac{1}{2}}\{f\}(\boldsymbol{x}),
\end{aligned}
$$

where $(-\triangle)^{-\frac{1}{2}}$ is the fractional integral operator with transfer function $\frac{1}{\|\boldsymbol{\omega}\|}$.

\subsection{First Derivative of the Radon Transform}

The relevant mathematical model for DPCI is based on the first derivative of the Radon transform with respect to the signed direction from the origin. It is denoted by

$$
\mathcal{R}_{\theta}^{(1)} f(y)=\frac{\partial}{\partial y} \mathcal{R}_{\theta} f(y)=\mathrm{D}_{y} \mathcal{R}_{\theta} f(y),
$$

where $\mathrm{D}_{y}$ denotes the partial derivative along the $y$ direction.

As a consequence of the linearity and the projected translation invariance properties of the Radon transform, FDRT is a linear operator and has itself the projected translation invariance property.

Proposition 1. Let $\mathcal{R}^{(1)^{*}}$ denote the adjoint of the FDRT. We have

$$
\mathcal{R}^{(1)^{*}} \mathcal{R}_{\theta}^{(1)}\{f\}(\boldsymbol{x})=2 \pi \times(-\triangle)^{\frac{1}{2}}\{f\}(\boldsymbol{x}),
$$

where $(-\triangle)^{\frac{1}{2}}$ is a fractional Laplace operator with transfer function $\|\boldsymbol{\omega}\|$.

Proof. Since $\mathrm{D}_{y}^{*}=\mathrm{D}_{y}$, we find that

$$
\mathcal{R}^{(1)^{*}} \mathcal{R}_{\theta}^{(1)}\{f\}(\boldsymbol{x})=\mathcal{R}^{*}\left\{-\mathrm{D}_{y}\right\} \mathrm{D}_{y} \mathcal{R}_{\theta}\{f\}(\boldsymbol{x}) .
$$

By using the Fourier-slice theorem and pseudo-commuting with the convolution property of the Radon transform, we have

$$
\mathcal{R}^{*}\left\{-\mathrm{D}_{y}\right\} \mathrm{D}_{y} \mathcal{R}_{\theta}\{f\}(\boldsymbol{x})=\mathcal{R}^{*} \mathcal{R}_{\theta}\{(-\triangle)\{f\}\}(\boldsymbol{x}) .
$$

The result then follows from (2).

\section{FAST AND ACCURATE IMPLEMENTATION OF FDRT}

In order to implement the FDRT, it is essential to define a discrete model that is consistent with the continuous domain and supports algebraic exactness and geometry fidelity.

\subsection{Discrete Model for FDRT}

We want to present the FDRT in the discrete domain. More precisely, the input is the discrete model of the object and the output is a set of sampled line integrals along a finite number of directions. These measurements should match the continuous-domain FDRT at the sampling positions. Let $\varphi$ denote a function that generates a Riesz basis of the space $\boldsymbol{V}$ as

$$
\boldsymbol{V}=\operatorname{span}\{\varphi(\cdot-\boldsymbol{k})\}_{\boldsymbol{k} \in \mathbb{Z}^{2}} .
$$

We define $c_{\boldsymbol{k}}=\langle f, \widetilde{\varphi}(\cdot-\boldsymbol{k})\rangle$, where $\widetilde{\varphi}$ is the dual of $\varphi$ with Fourier transform $\widehat{\widetilde{\varphi}}(\boldsymbol{\omega})=\frac{\widehat{\varphi}(\boldsymbol{\omega})}{\sum_{\boldsymbol{k} \in \mathbb{Z}^{2}}|\widehat{\varphi}(\boldsymbol{\omega}-2 \pi k)|^{2}}$. The projection of the object $f$ on the space $\boldsymbol{V}$ is

$$
P_{V} f(\boldsymbol{x})=\sum_{\boldsymbol{k} \in \mathbb{Z}^{2}} c_{k} \varphi_{\boldsymbol{k}}(\boldsymbol{x})
$$

where $\varphi_{\boldsymbol{k}}(\boldsymbol{x})=\varphi(\boldsymbol{x}-\boldsymbol{k})$.

Using the linearity and translation invariance properties of the FDRT, we have

$$
\mathcal{R}_{\theta}^{(1)}\left\{P_{V} f\right\}(y)=\sum_{\boldsymbol{k} \in \mathbb{Z}^{2}} c_{\boldsymbol{k}} \mathcal{R}_{\theta}^{(1)} \varphi_{\boldsymbol{k}}(y)
$$

where

$$
\mathcal{R}_{\theta}^{(1)} \varphi_{\boldsymbol{k}}(y)=\mathcal{R}_{\theta}^{(1)} \varphi(y-\langle\boldsymbol{\theta}, \boldsymbol{k}\rangle) .
$$

With regard to (4), the problem is reduced to finding an appropriate generating function and to determining analytically its FDRT. In this work, we use a tensor-product B-spline which provides a costquality tradeoff, where cost and quality are related to the size and the order of the generating function, respectively. Specifically we set

$$
\varphi(\boldsymbol{x})=\beta^{m}(\boldsymbol{x})=\beta^{m}\left(x_{1}\right) \beta^{m}\left(x_{2}\right),
$$


where $\beta^{m}\left(x_{i}\right), i=1,2$ is the centered univariate B-spline function of degree $m$.

Proposition 2. The FDRT of the tensor-product B-spline $\beta^{m}(\boldsymbol{x})=$ $\beta^{m}\left(x_{1}\right) \beta^{m}\left(x_{2}\right)$ is given by

$$
\mathcal{R}_{\theta}^{(1)}\left\{\beta^{m}\right\}(y)=\frac{\triangle_{|\cos \theta|}^{m+1} \triangle_{|\sin \theta|}^{m+1}}{(2 m) !} y_{+}^{2 m},
$$

where $\triangle_{h}^{n} f(y)$ is the $n$-fold iteration of the finite-difference operator $\triangle_{h} f(y)=\frac{f(y)-f(y-h)}{h}$.

Proof. We define $g_{\theta}(y)=\mathcal{R}_{\theta}^{(1)}\left\{\beta^{m}\right\}(y)$. By an application of the Fourier-slice theorem, we have

$$
g_{\theta}(y)=\mathcal{F}^{-1}\left\{j \omega \widehat{\beta^{m}}(\omega \cos \theta) \widehat{\beta^{m}}(\omega \sin \theta)\right\},
$$

where $\widehat{\beta^{m}}(\omega)$ is the Fourier transform of $\beta^{m}(y)$. So,

$$
\begin{aligned}
g_{\theta}(y) & =\frac{\partial}{\partial y}\left(\triangle_{|\cos \theta|}^{m+1} \frac{y_{+}^{m}}{m !}\right) *\left(\triangle_{|\sin \theta|}^{m+1} \frac{y_{+}^{m}}{m !}\right) \\
& =\frac{\triangle_{|\cos \theta|}^{m+1} \triangle_{|\sin \theta|}^{m+1}}{(2 m) !} y_{+}^{2 m} .
\end{aligned}
$$

In our formulation, we represent the object by the B-spline expansion

$$
f(\boldsymbol{x})=\sum_{\boldsymbol{k} \in \mathbb{Z}^{2}} c_{\boldsymbol{k}} \varphi(\boldsymbol{x}-\boldsymbol{k})
$$

which is fully specified by its B-spline coefficients $c_{k}$. To discretize the reconstruction problem and specify our forward model, we need to calculate the FDRT of $f$ at the location $\left(y_{j} ; \theta_{i}\right)$, where $y_{j}=j \Delta y$ and $\theta_{i}=i \Delta \theta$. Specifically, the discrete model of the FDRT of $f$ is

$$
\mathcal{R}_{\theta_{i}}^{(1)} f[j]=\sum_{\boldsymbol{k} \in \mathbb{Z}^{2}} c_{\boldsymbol{k}} \mathcal{R}_{\theta_{i}}^{(1)} \varphi_{\boldsymbol{k}}\left(y_{j}\right) .
$$

The linear weights $\mathcal{R}_{\theta_{i}}^{(1)} \varphi_{\boldsymbol{k}}\left(y_{j}\right)$ can be calculated using (5).

\subsection{Matrix Formulation of the Forward Model}

To specify the forward imaging model, we now introduce an equivalent matrix formulation of (7) which reads

$$
\mathrm{g}=\mathbf{H c},
$$

where $\mathbf{c}$ is a vector of B-spline coefficients in lexical order with $\mathbf{c}(\boldsymbol{k})=c_{\boldsymbol{k}}$, where $\mathbf{g}$ is the output vector, and whose $\mathbf{H}$ is the system matrix with

$$
[\mathbf{H}]_{(i, j), \boldsymbol{k}}=\mathcal{R}_{\theta_{i}}^{(1)} \varphi_{\boldsymbol{k}}\left(y_{j}\right) .
$$

The matrix entries $\mathcal{R}_{\theta_{i}}^{(1)} \varphi_{\boldsymbol{k}}\left(y_{j}\right)$ are calculated using (5). The corresponding adjoint operator is represented by the conjugate transpose of the system matrix.

In our implementation, we use cubic B-spline basis functions and take advantage of the closed-form (6). For better efficiency, these projection functions are stored in a lookup table. Since we present an exact discrete model for the FDRT, our implementation is accurate-using a lookup table and multi-threading makes it fast.

\section{RECONSTRUCTION METHODS}

For large images with a limited number of projections, direct solution methods such as FBP are not accurate enough. In this case, iterative techniques provide much more robust means of solving the underlying large-scale system of equations.

In our implementation, we take advantage of our forward-model algorithm. Using the symbol $\mathbf{H}$ as a generic system matrix, the cost function that we want to minimize with respect to (8) is

$$
J(\mathbf{c})=\frac{1}{2}\|\mathbf{H} \mathbf{c}-\mathbf{g}\|^{2} .
$$

Since image reconstruction with a limited number of projections is ill-posed, we introduce a combination of anisotropic discrete TV and Tikhonov regularization. We then aim at finding a vector $\mathbf{c}$ that minimizes the cost function

$$
J(\mathbf{c})=\frac{1}{2}\|\mathbf{H} \mathbf{c}-\mathbf{g}\|^{2}+\frac{\lambda_{1}}{2}\|\mathbf{c}\|^{2}+\lambda_{2} \sum_{i}\left\|\{\mathbf{L} \mathbf{c}\}_{i}\right\|_{1} .
$$

where $\{\mathbf{L c}\}_{i} \in \mathbb{R}^{2}$ is a vector containing a finite difference in position $\mathbf{c}_{i}$ along horizontal and vertical directions. The summation is on all image B-spline coefficients.

We reformulate the TV problem (10) using variable splitting as a linear equality-constrained problem. We form its augmentedLagrangian function as

$$
\begin{aligned}
\mathcal{L}_{\mu}(\mathbf{c}, \mathbf{u}, \boldsymbol{\alpha})= & \frac{1}{2}\|\mathbf{H} \mathbf{c}-\mathbf{g}\|^{2}+\frac{\lambda_{1}}{2}\|\mathbf{c}\|^{2}+\lambda_{2} \sum_{i}\left\|\mathbf{u}_{i}\right\|_{1} \\
& +\boldsymbol{\alpha}^{T}(\mathbf{L} \mathbf{c}-\mathbf{u})+\frac{\mu}{2}\|\mathbf{L} \mathbf{c}-\mathbf{u}\|^{2}
\end{aligned}
$$

where $\boldsymbol{\alpha} \in \mathbb{R}^{2 \times N^{2}}$ is the vector of Lagrange multipliers and $N^{2}$ is the image size. To minimize (11), we apply the ADMM to separate the problem in two basic optimization problems with respect to a single variable, which consists of the iterations

$$
\left\{\begin{array}{l}
\mathbf{c}^{k+1} \leftarrow \underset{\mathbf{c}}{\operatorname{argmin}} \mathcal{L}_{\mu}\left(\mathbf{c}, \mathbf{u}^{k}, \boldsymbol{\alpha}^{k}\right) \\
\mathbf{u}^{k+1} \leftarrow \underset{\mathbf{u}}{\operatorname{argmin}} \mathcal{L}_{\mu}\left(\mathbf{c}^{k+1}, \mathbf{u}, \boldsymbol{\alpha}^{k}\right) \\
\boldsymbol{\alpha}^{k+1} \leftarrow \boldsymbol{\alpha}^{k}+\mu\left(\mathbf{L} \mathbf{c}^{k+1}-\mathbf{u}^{k+1}\right) .
\end{array}\right.
$$

$\mathcal{L}_{\mu}\left(\mathbf{c}, \mathbf{u}^{k}, \boldsymbol{\alpha}^{k}\right)$ is a quadratic function with respect to $\mathbf{c}$ whose gradient is

$$
\begin{aligned}
\nabla \mathcal{L}_{\mu}\left(\mathbf{c}, \mathbf{u}^{k}, \boldsymbol{\alpha}^{k}\right)= & \left(\mathbf{H}^{H} \mathbf{H}+\mu \mathbf{L}^{H} \mathbf{L}+\lambda_{1} \mathrm{I}\right) \mathbf{c} \\
& -\left(\mathbf{H}^{H} \mathbf{g}+\mu \mathbf{L}^{H}\left(\mathbf{u}^{k}-\frac{\boldsymbol{\alpha}^{k}}{\mu}\right)\right) .
\end{aligned}
$$

We minimize $\mathcal{L}_{\mu}\left(\mathbf{c}, \mathbf{u}^{k}, \boldsymbol{\alpha}^{k}\right)$ iteratively using the CG algorithm. Since $\mathbf{H}^{H} \mathbf{H}$ has a large condition number, we need to find a transformation that produces a condition number as close to 1 as possible to improve the rate of convergence. We call the matrix $\mathbf{M}$ a preconditioner. It is such that $\mathbf{M}^{-1}\left(\mathbf{H}^{H} \mathbf{H}+\mu \mathbf{L}^{H} \mathbf{L}+\lambda_{1} \mathrm{I}\right)$ has a formable set of eigenvalues. In principle, we can compute a selfadjoint, positive square root of $\mathbf{M}$, which we denote by $\mathbf{M}^{\frac{1}{2}}$.

From the continuous-domain formulation (3) of our our problem, we know that $\mathcal{R}^{(1)^{*}} \mathcal{R}^{(1)}$ has a Fourier transform that is proportional to $\|\boldsymbol{\omega}\|$ and $\mathbf{L}^{H} \mathbf{L}$ is a Laplace operator. Thus, the preconditioner $\mathbf{M}^{-1}$ that we use in the discrete domain is the discrete filter whose frequency response is $\frac{1}{\|\boldsymbol{\omega}\|+\mu\|\boldsymbol{\omega}\|^{2}+\lambda_{1}}$. 
It is easy to show that the solution of the minimization of $\mathcal{L}_{\mu}\left(\mathbf{c}^{k}, \mathbf{u}, \boldsymbol{\alpha}^{k}\right)$ with respect to $\mathbf{u}$ is given by the shrinkage

$$
\mathbf{u}^{k+1}=\max \left\{\left|\mathbf{L} \mathbf{c}^{k+1}+\frac{\boldsymbol{\alpha}^{k}}{\mu}\right|-\frac{\lambda_{2}}{\mu}, 0\right\} \operatorname{sgn}\left(\mathbf{L} \mathbf{c}^{k+1}+\frac{\boldsymbol{\alpha}^{k}}{\mu}\right) .
$$

\section{EXPERIMENTAL RESULTS}

As the original object, we use the realistic analytical brain phantom [8] shown in Figure 2(a). It has size $256 \times 256$. Starting from the B-spline coefficients of the object, we simulate the DPCI acquisition by applying the forward model (8).

We investigated the performance of the direct method and the ADMM algorithm by using our proposed FDRT implementation to solve the inverse problem (8). Our simulation results show that using the proposed preconditioner improves the convergence speed ten fold in comparison with the conventional CG method. The signalto-noise ratio (SNR) of the images reconstructed with only 60 projections using the ADMM and FBP approach are $13.4 \mathrm{~dB}$ and $5.7 \mathrm{~dB}$, respectively. The reconstruction results are displayed in Figures 2(c) and 2(d). To get a result as good as in Figure. 2(c), the direct method requires 180 projections, which is three times more data and as much increase in acquisition time.

We also compared the proposed ADMM method with two standard methods for the solution of TV-regularized inverse problems: iterative shrinkage thresholding algorithm (ISTA) and FISTA [9] in Figure 2(b). The required time to reach the value $4 \times 10^{7}$ of the objective function is $11 \mathrm{~s}$ and $54 \mathrm{~s}$ for ADMM and FISTA, respectively. This suggests that our method is about 4 times faster than FISTA, which is often presented as the state-of-the-art technique for solving TV-regularization problems.

\section{CONCLUSION}

We presented an exact B-spline formulation for the discretization of the first derivative of the Radon transform that avoids any numerical differentiation in 2-D. This result can be easily extended to higher dimensions. We introduced a new algorithm that uses the alternating direction method of multipliers to solve the TV-regularized reconstruction problem of DPCI. An important practical twist is the introduction of a problem-specific preconditioner which significantly speeds up the quadratic optimization step of the algorithm. Our simulation results suggest that the one ADMM method is competitive with state-of-the-art methods to solve the TV-reconstruction problem.

Acknowledgements: The authors would like to thank Prof. Marco Stampanoni and Dr. Peter Modregger from the Swiss Light Source (PSI) for their support and helpful discussions.

\section{References}

[1] J. Zambelli, N. Bevins, Z. Qi, and G. Chen, "Radiation dose efficiency comparison between differential phase contrast CT and conventional absorption CT," Medical Physics Letter, vol. 37, pp. 2473-2479, 2010.

[2] F. Pfieffer, O. Bunk, C. Kottler, and C. David, "Tomographic reconstruction of three-dimensional objects from hard X-ray differential phase contrast projection images," Nuclear Instrument and Methods in Physics Research, pp. 925-928, 2007.

[3] M. Strobl, W. Treimer, P. Walter, S. Keil, and I. Manke, "Magnetic field induced differential neutron phase contrast imaging," Applied Physics Letter, vol. 91, pp. 569-587, 2007.
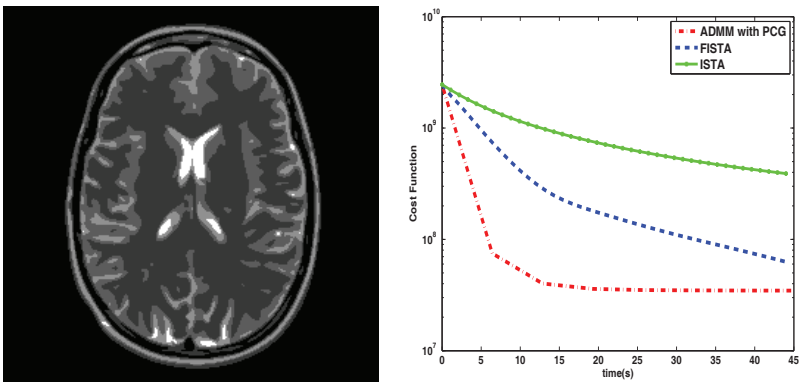

(a) Phantom.

(b) Comparison of different TVreconstruction methods

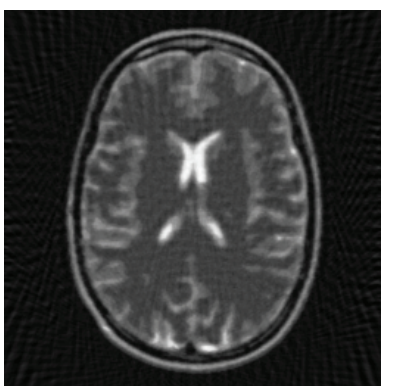

(c) ADMM with PCG, 60 projec- (d) tions, $\mathrm{SNR}=14.7 \mathrm{~dB}$.

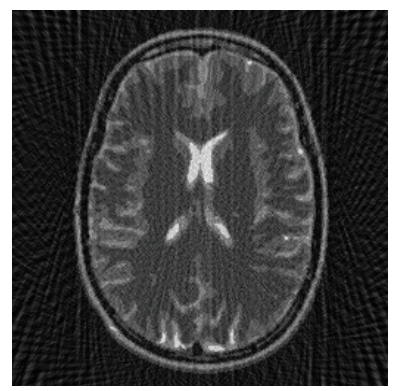

FBP,

60 projections,
Fig. 2: Experimental results.

[4] Z. Qi, J. Zambelli, N. Bevins, and G. Chen, "A novel method to reduce data acquisition time in differential phase contrast computed tomography using compressed sensing," Proceedings of SPIE, vol. 7258, pp. 4A1-8, 2009.

[5] T. Kohler, B. Brendel, and E. Roessl, "Iterative reconstruction for differential phase contrast imaging using spherically symmetric basis functions," Medical Physics Letter, vol. 38, pp. 4542-4545, 2011.

[6] M. Unser, "Sampling-50 years after Shannon," Proceedings IEEE, vol. 88, pp. 254104-1-3, 2000.

[7] Frank Natterer, The Mathematics of Computed Tomography, John Wiley and sons, 1986.

[8] M. Guerquin-Kern, L. Lejeune, K. P. Pruessmann, and M. Unser, "Realistic analytical phantoms for parallel magnetic resonance imaging," IEEE Transactions on Medical Imaging, 2012, 10.1109/ISBI.2010.5490365.

[9] A. Beck and M. Teboulle, "A fast iterative shrinkagethresholding algorithm for linear inverse problems," SIAM imaging sciences, vol. 2, pp. 183-202, 2009. 